\title{
SVM-Based Sleep Apnea Identification Using Optimal RR-Interval Features of the ECG Signal
}

\author{
Laiali Almazaydeh ${ }^{1}$, Khaled Elleithy ${ }^{2}$, Miad Faezipour ${ }^{3}$, Helen Ocbagabir ${ }^{4}$
}

Accepted 15 th August 2014

DOI: 10.18201/ijisae.79075

\begin{abstract}
Sleep apnea (SA) is the most commonly known sleeping disorder characterized by pauses of airflow to the lungs and often results in day and night time symptoms such as impaired concentration, depression, memory loss, snoring, nocturnal arousals, sweating and restless sleep. Obstructive Sleep Apnea (OSA), the most common SA, is a result of a collapsed upper respiratory airway, which is majorly undiagnosed due to the inconvenient Polysomnography (PSG) testing procedure at sleep labs. This paper introduces an automated approach towards identifying sleep apnea. The idea is based on efficient feature extraction of the electrocardiogram (ECG) signal by employing a hybrid of signal processing techniques and classification using a linear-kernel Support Vector Machine (SVM). The optimum set of RR-interval features of the ECG signal yields a high classification accuracy of $97.1 \%$ when tested on the Physionet Apnea-ECG recordings. The results provide motivating insights towards future developments of convenient and effective OSA screening setups.
\end{abstract}

Keywords: sleep apnea, PSG, ECG, RR interval, features extraction, SVMs.

\section{Introduction}

Sleep is the circadian rhythm which is among the most crucial needs in our day to day activities. On average, humans spend approximately one-third of their lifespan sleeping. Getting enough hours of sleep indicate repaired blood pressure, heart rate, and relaxed muscles and tissues [1].

A sleeping disorder occurs when one cannot sleep and has symptoms like excessive daytime sleepiness and fatigue. Sleep Apnea (SA) is among the very common respiratory sleeping disorders characterized by cessations of airflow to the lungs or having a very low breath. The cessations lasting in more than 10 seconds considered as apnea event might occur 5 to 30 times in an hour and up to 400 per night [2]. Clinically, sleep apnea is divided into Obstructive Sleep Apnea (OSA) and Central Sleep Apnea (CSA). OSA, being the most common SA, is generally caused by a collapse of the upper respiratory airway. CSA is a neurological condition where brain fails to appropriately control breathing [3] [4].

Statistics show that out of 18 million Americans suffering from OSA, around 10 million remain undiagnosed [5]. The undiagnosed cases are due to inconvenience, expenses and unavailability of testing. The Polysomnography (PSG) is the current and traditional testing process which is a standard procedure ordered for all sleep disorders. This testing records the breath airflow, respiratory movement, oxygen saturation, body saturation, body position, electroencephalogram (EEG), electroculogram (EOG), electromyogram (EMG), and

\footnotetext{
${ }^{1}$ Department of Software Engineering, Al Hussein Bin Talal University, Jordan

2,3,4 Department of Computer Science and Engineering, University of Bridgeport, CT 06604, USA

* Corresponding Author:email: lalmazay@my.bridgeport.edu

\# This paper has been presented at the International Conference on

Advanced Technology\&Sciences (ICAT'14) held in Antalya (Turkey), August 12-15, 2014
}

This journal is @ Advanced Technology \& Science 2013 electrocardiogram (ECG) to determine the sleep stages [6].

To summarize, PSG needs to be replaced by more convenient detection methods and faster treatment. In this regard, we present the fully automated identification of the apnea periods based on Support Vector Machines (SVMs) using the RR-interbeat interval series in ECG signal that will be used in a larger real time system for SA diagnosis. The objective of the system is to alert a patient

who might be subject to an apnea attack.

This paper is organized as follows. In Section II, we glance at a variety of sleep apnea detection methods. In section III, the methodology of our proposed system is described. Section IV demonstrates the results of our system. Finally, we conclude our paper in section $\mathrm{V}$, and highlight some directions for future research.

\section{Related Works}

Over the past few years, most of the related research has focused on detecting OSA through statistical features of different signals such as thorax and abdomen effort signals, nasal air flow, oxygen saturation, electrical activity of the heart (ECG), and electrical activity of the brain (EEG).

In our previous published research, we developed a Neural Network (NN) as a predictive tool for OSA using oxygen saturation signal (SpO2) measurements obtained from pulse oximetry [7].

Many studies perform detection of OSA through heart rate variability (HRV) and the ECG signal. Quiceno-Manrique et al. [8] proposed a simple diagnostic tool for OSA with a high accuracy (up to $92.67 \%$ ) using time-frequency distributions and dynamic features in ECG signal. Moreover, based on spectral components of heart rate variability, frequency analysis was performed in [9] using Fourier and Wavelet Transformation with appropriate application of the Hilbert Transform, where the sensitivity was $90.8 \%$. In addition, in [10] a bivariate 
autoregressive model was used to evaluate beat-by-beat power spectral density of HRV and R peak area, where the classification results showed accuracy higher than $85 \%$.

During periods of prolonged OSA, cyclic increases and decreases of heart rate are typically associated with the apneic phase and the resumption of breathing [11]. Therefore, the technique in this work also relies on features of the ECG signal to detect and quantify these periods of OSA by the fully automated identification of these dynamic features in the RR-interbeat interval series based on the ones suggested by Chazal et al. [6], and Yilmaz et al. [12].

\section{Proposed Methodology}

In this work, the overall design involves acquiring the ECG signal. This signal is then processed to cancel the noise and detect RR-interval. Then, a combination of the most effective set of RRinterval based features of the ECG signal is calculated for classification. In what follows, our detection system design is described and shown in Figure 1.

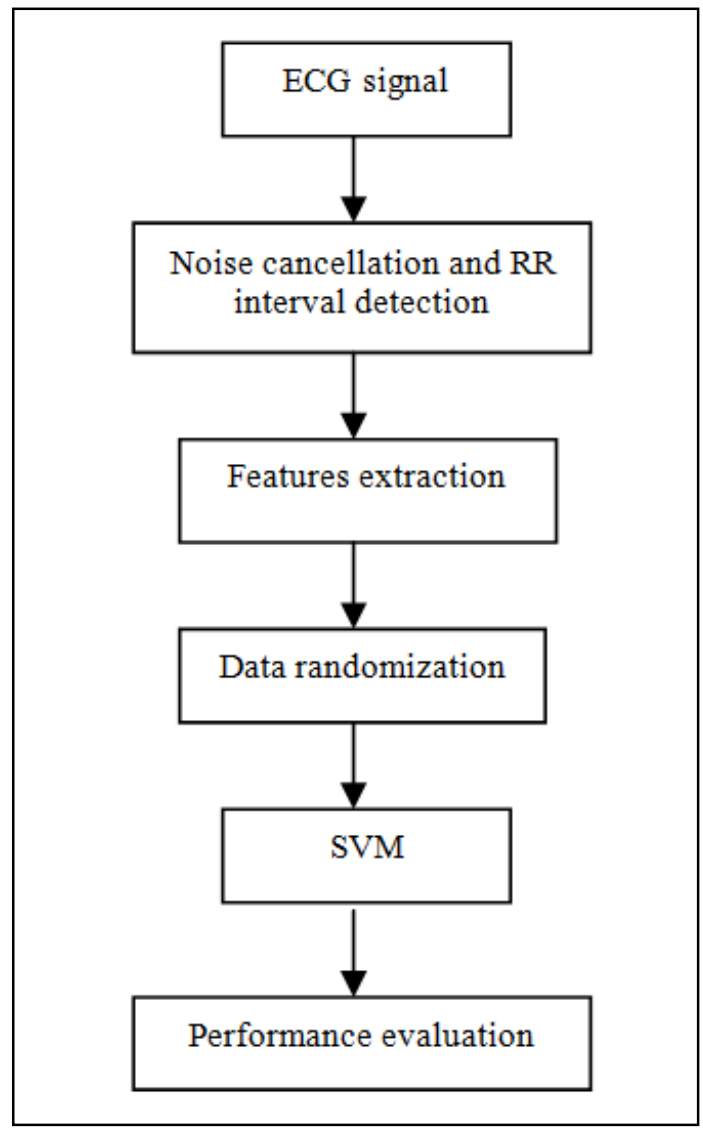

Figure 1. Schematic diagram of the system

\subsection{Database}

In this work, the overall design involves acquiring the ECG signal. This signal is then processed to cancel the noise and detect RR-interval. Then, a combination of the most effective set of RRinterval based features of the ECG signal is calculated for classification. In what follows, our detection system design is described and shown in Figure 1.

A. Database

The database used in this study is available from the PhysioNet web site [13]. The Apnea-ECG Database contains 70 recordings, containing a single continuous ECG signal varying in length of approximately 8 hours duration. The sampling frequency of ECG signal is $100 \mathrm{~Hz}$, with 16-bit resolution, with one sample bit representing $5 \mu \mathrm{V}[6]$.

The database was scored by clinical experts by dividing the recordings into a set of one-minute segments. The segments were classified as "apnea", if at any time during that minute there was evidence of SA on the basis of respiration and oxygen saturation. Otherwise, it was classified as "normal" [6].

\subsection{Noise cancellation and $R$ Wave detection}

SA episodes consist of bradycardia during apnea followed by tachycardia upon its cessation, which represent cyclic variations in the duration of a heartbeat, also known as RR intervals of ECG signal [6].

Generally, the ECG sleep apnea recognition techniques used have two parts: characteristics (or features) extraction, and waveform classification and recognition [14].

The characteristics extraction includes noise cancellation and QRS complex wave detection. The R-wave which has the highest (or lowest) value in the QRS complex wave is the outstanding characteristic of the ECG signal.

The R-wave detection technique used in this paper is a modified version of the traditional "Pan and Tompkins" algorithm [15] with the use of adaptive filters in noise cancellation. The whole algorithm is divided into five-steps; noise cancellation using adaptive filtering, signal slope detection, squaring, windowing, and RR-wave interval calculation.

1) Adaptive Filtering: The Least Mean Square adaptive algorithm is one of the most robust techniques used to reduce any random noise signal interfaced to the ECG. A step size of $0.8 \times 10-5$ and filter length of 106 can be used to cancel any noise added to the recorded ECG.

2) Signal Slope Detection: A differentiator is used to compute the QRS complex slope waveform information. A five point derivative is used with the following transfer function:

$H(z)=(1 / 8 T)\left(-z^{-2}-2 z^{-1}+2 z^{1}+Z^{2}\right)$

The difference equation is:

$y(n T)=(1 / 8 T)[-x(n T-2 T)-2 x(n T-T)+2 x(n T+T)+x(n T+2 T)]$

3) Squaring: The point by point squaring function is described by the following equation:

$$
y(n T)=[x(n T)]^{2}
$$

This results in positive data points and also performs nonlinear amplification of the differentiated ECG frequencies.

4) Windowing: Additional waveform features are calculated by a moving-window integration equation given by:

$y(n T)=(1 / N)[x(n T-(N-1) T)+x(n T-$

$(N-2) T) \ldots .+x(n T)]$

Where $\mathrm{N}$ is the number of samples in the width of the integration window. For a sample rate of 200, window of 30 samples wide...

5) RR wave interval calculation: Automatically adjustable thresholds to float over the noise are applied to the integrated wave form. The applied set of thresholds are calculated from:

$S P K=0.125 P E A K+0.875 S P K$

(if PEAK is the signal peak)

$N P K=0.125 P E A K+0.875 N P K$

(if PEAK is the noise peak)

THRESHOLD $=N P K+0.25(S P K-N P K)$

THRESHOLD $=0.5 T H R E S H O L D$ 
In the above equations, all the variables refer to the integration waveform: PEAK is the overall peak, SPK is the running estimate of the signal peak, NPK is the running estimate of the noise peak, THRESHOLD1 is the first threshold applies, and THRESHOLD2 is the second threshold applied. Every time a peak is recognized, a QRS complex is identified in the filtered and integrated waveform. The RR average is then given by taking the mean of the eight most recent consecutive RR intervals.

$$
R R_{A V E R A G E}=0.125\left(R R_{n-7}+R R_{n-6}+\ldots+R R_{n}\right)(9)
$$

\subsection{Feature Extraction}

Our technique relies on a large set of an effective combination of ECG signal features. These features could potentially be used for classification. The features considered are a novel hybrid of features extracted from [6] and [12]. The following are the most effective set of RR-interval based features of the ECG signal for apnea detection:

- Mean epoch and recording RR-interval.

- Standard deviation of the epoch and recording RR-interval.

- The NN50 measure (variant 1), defined as the number of pairs of adjacent RR-intervals where the first RR-interval exceeds the second RR-interval by more than $50 \mathrm{~ms}$.

- The NN50 measure (variant 2), defined as the number of pairs of adjacent RR-intervals where the second RR-interval exceeds the first RR interval by more than $50 \mathrm{~ms}$.

- Two pNN50 measures, defined as each NN50 measure divided by the total number of RR-intervals.

- The SDSD measures, defined as the standard deviation of the differences between adjacent RR-intervals.

- The RMSSD measures, defined as the square root of the mean of the sum of the squares of differences between adjacent RR-intervals.

- Median of RR-intervals.

- Inter-quartile range, defined as difference between 75th and 25th percentiles of the RR-interval value distribution.

- Mean absolute deviation values, defined as mean of absolute values obtained by the subtraction of the mean RR-interval values from all the RR-interval values in an epoch.

\subsection{Data Randomization}

Since apnea is defined as a pause in breathing, and can last from a few seconds to minutes (almost $>=10 \mathrm{sec}$ ), we investigate and analyze three cases of data partitioning to determine the best accuracy that can be achieved. The apnea and regular data are partitioned into $10 \mathrm{sec}, 15 \mathrm{sec}$, and $30 \mathrm{sec}$ pieces. In this step, we separate the training data and testing data with $80 \%$ for training and $20 \%$ for testing. A MATLAB built-in function (rand) is used to determine whether a feature set in $10 \mathrm{sec}, 15 \mathrm{sec}$ or $30 \mathrm{sec}$ of data belongs to test group or rule creation group. After the signals are separated, we perform the training for SVMs.

Table 1.10 sec. (Accuracy is $86.1 \%$ )

\begin{tabular}{|c|l|l|}
\hline \multicolumn{1}{|c|}{ Tnut|Output } & Regular & Apnea \\
\hline Regular & $\mathbf{9 7 . 2 \%}$ & $2.78 \%$ \\
\hline Apnea & $25 \%$ & $\mathbf{7 5 \%}$ \\
\hline
\end{tabular}

Table 2.15 sec. (Accuracy is $96.5 \%$ )

\begin{tabular}{|c|c|c|}
\hline Input\Output & Regular & Apnea \\
\hline Regular & $\mathbf{1 0 0 \%}$ & $0 \%$ \\
\hline Apnea & $7.1 \%$ & $\mathbf{9 2 . 9 \%}$ \\
\hline
\end{tabular}

Table $3.30 \mathrm{sec}$. (Accuracy is $95 \%$ )

\begin{tabular}{|c|c|c|}
\hline Input Output & Regular & Apnea \\
\hline Regular & $\mathbf{1 0 0 \%}$ & $0 \%$ \\
\hline Apnea & $10 \%$ & $\mathbf{9 0 \%}$ \\
\hline
\end{tabular}

\subsection{Data Randomization}

In order to investigate apneic epoch detection, we use SVMs as a classification method. SVM is one of a powerful machine learning technique from supervised learning category, which used as a training algorithm for analyzing data and recognizing patterns. When we have two classes of data, SVM performs classification by building a maximal margin hyperplane that optimally separates the data into two groups. In general, the larger the margin is, the lower the generalization error of the classifier. SVM handles the separation by using a kernel function to map the data into a different space with a hyperplane. There are many kernels available for SVM, which provides flexibility for the constructed hyperplane to partition the data [4]. In our implementation, we use a linear kernel function to map the training data into kernel space. In the optimization process, we use a method called sequential minimal optimization to find the separating hyperplane.be

\section{Results}

To build our model, we used MATLAB toolset. The data records were imported as MATLAB matrices (.mat) from physionet web site. We evaluated the effectiveness of our model on the Apnea ECG database, using different records available in that database. We ran two scenarios in our experiment: the whole combination of the features, and a combination of every two separate features.

\subsection{All Features}

To evaluate the performance of the classification system, two statistical indicators, Sensitivity (Se) and Specificity (Sp) in addition to the Accuracy (Acc) have been used. The sensitivity of a test is the percentage of patients in the OSA positive group correctly diagnosed, whereas the specificity is the percentage of subjects in the OSA negative group correctly classified by the test.

Tables I, II and III show the classification results with all extracted features for the three cases mentioned in the data partitioning step. Our model was based on a linear kernel SVM using set of RR-interval features of the ECG signal. The three cases used here are: (i) $10 \mathrm{sec}$, (ii) $15 \mathrm{sec}$, and (iii) $30 \mathrm{sec}$. The accuracy of our approach is $86.1 \%, 96.5 \%$, and $95 \%$, respectively. From Table II, SVM with linear kernel using 15 second epochs shows the best classification accuracy with high successful rate of correct prediction.

\subsection{Every Two Separate Features}

The idea here is to reduce the feature set for classification for less processing, while simultaneously maintaining high classification accuracy. In this part of analysis, the $15 \mathrm{sec}$ data are used. The analysis randomly selected $80 \%$ of normal data and randomly selected $20 \%$ of apnea data. The program has a for loop to run the training and classification process for 10 times to take the average value.

Table IV shows the classification accuracy results for the combination set of every two features. From the table we can conclude that the best two feature-combinations are 25 and 75 percentiles of RR interval along with the mean absolute value 
Table 4. Classification accuracy of the combination of every two different features.

\begin{tabular}{|c|c|c|c|c|c|c|c|c|c|c|c|}
\hline Mean value & & 70.4 & 59.4 & 52.1 & 559 & 55.1 & 739 & 65.6 & 643 & 93.6 & 90.6 \\
\hline Standard deviation & 69.0 & & 63.7 & 65.1 & 45.7 & 539 & 50.6 & 603 & 643 & 96.8 & 91.8 \\
\hline NN50 variant 1 & 57.4 & 633 & & 559 & 60.4 & 62.7 & 62.0 & 59.8 & 59.4 & 93.7 & 89.7 \\
\hline NN50 variant2 & 57.8 & 64.0 & 57.8 & & 613 & 56.4 & 61.6 & 603 & 59.1 & 948 & 90.8 \\
\hline SDSD & 71.1 & 46.8 & 61.7 & 61.4 & 49.4 & 49.5 & & 60.4 & 67.4 & 95.0 & 91.0 \\
\hline RMSSD & 61.6 & 57.8 & 58.0 & 61.6 & 479 & 49.8 & 592 & & 669 & 96.1 & 90.1 \\
\hline Median of & 60.7 & 65.1 & 56.0 & 62.6 & 593 & 545 & 673 & 619 & & 872 & 912 \\
\hline 25 and 75 percentiles & 949 & 96.7 & 952 & 94.6 & 95.0 & 912 & 942 & 96.8 & 88.6 & & 97.1 \\
\hline Mean absolute value & 93.1 & 82.3 & 95.1 & 90.1 & 88.1 & 87.0 & 92.6 & 902 & 91.6 & 93.1 & \\
\hline
\end{tabular}

\section{Conclusion}

In this work, we studied the possibility of the detection of SA events from the ECG signal variation patterns. We evaluated the effectiveness of our model on the Apnea ECG database, using different records available in that database.

Our model was based on a selective set of RR-interval based features that were given to SVM for classification. We evaluated our model on three different epoch lengths and different combination of two-features set scenarios. From the experimental results, we conclude that SVM with linear kernel shows the best accuracy with 15 second epoch length. Two optimum features were also derived from statistical analysis. A future direction to this work would be incorporating this work into a real time monitoring system that acquires and analyzes the ECG signal of subjects during sleep.

\section{References}

[1] W. Mendelson, Human Sleep: Research and Clinical Care. Plenum Medical Book Company, New York and London.

[2] Sleep Disorders Guide. www.sleepdisorderguide.com.

[3] S. Isa, M. Fanany, W. Jatmiko and A. Arymurthy, "Sleep Apnea Detection from ECG Signal, Analysis on Optimal Features, Principal Components, and Nonlinearity," in Proceedings of the 5th IEEE International Conference on Bioinformatics and Biomedical Engineering (iCBBE), pp. 1-4, May 2011.

[4] L. Almazaydeh, K. Elleithy, and M. Faezipour, "Detection of obstructive Sleep Apnea through ECG Signal Features," in Proceedings of the 2012 IEEE International Conference on Electro Information Technology (IEEE eit2012), pp. 1-6, May 2012.

[5] SleepMedInc. www.sleepmed.md.

[6] P. Chazal, T. Penzel and C. Heneghan, "Automated Detection of Obstructive Sleep at Different Time Scales Using the Electrocardiogram," Institute of Physics Publishing, Vol. 25, no. 4, pp. 967-983, Aug 2004.

[7] L. Almazaydeh, M. Faezipour, and K. Elleithy," A Neural Network System for Detection of Obstructive
Sleep Apnea through SpO2 Signal fFeatures," in International Journal of Advanced Computer Science and Applications, (IJACSA), vol. 3, no. 5, pp. 7-11, Jun. 2012.

[8] Q. Manrique, A. Hernandez, T. Gonzalez, F. Pallester and C. Dominquez," Detection of Obstructive Sleep Apnea in ECG Recordings Using Time-Frequency Distributions and Dynamic Features," in Proceedings of the IEEE International Conference on Engineering in Medicine and Biology Society (EMBS 2009), pp. 55595562, Sep. 2009.

[9] M. Schrader, C. Zywietz, V. Einem, B. Widiger, G. Joseph, "Detection of Sleep Apnea in Single Channel ECGs from the PhysioNet Data Base," Computers in Cardiology 2000, vol. 27, pp. 263-266, Sep. 2000.

[10] M. Mendez, D. Ruini, O. Villantieri, M. Matteucci, T. Penzel and A. Bianchi, "Detection of Sleep Apnea from Surface ECG Based on Features Extracted by an Autoregressive Model, " in Proceedings of the IEEE International Conference on Engineering in Medicine and Biology Society (EMBS 2007), pp. 6105-6108, Aug. 2007.

[11] JE. Mietus, CK. Peng, P Ch. Ivanov and AL. Goldberger, "Detection of Obstructive Sleep Apnea from Cardiac Interbeat Interval Time Series," Computers in Cardiology 2000, vol. 27, pp. 753-756, 2000.

[12] B. Yilmaz, M. Asyali, E. Arikan, S. Yektin and F. Ozgen, "Sleep Stage and Obstructive Apneaic Epoch Classification Using Single Lead ECG," in Biomedical Engineering Online, vol. 9, 2010.

[13] PhysioNet, www.physionet.org.

[14] R. Lee, I. Chou, C. Lai, M. Liu and M. Chiu, "A Novel QRS Detection Algorithm Applied to the Analysis for Heart Rate Variability of Patients with Sleep Apnea," in Biomedical Engineering Applications, Basis \& Communications, vol. 17, no. 5, pp. 44-48, Oct. 2005.

[15] J. Pan and W. Tompkins, "A Real-Time QRS Detection Algorithm," in IEEE Transactions on Biomedical Engineering, vol. BME-32, no. 3, pp. 230-236, Mar. 1985. 